\title{
The Interstellar Radiation Field of the Milky Way in Three Spatial Dimensions
}

\author{
Troy A. Porter* \\ W. W. Hansen Experimental Physics Laboratory and Kavli Institute for Particle Astrophysics and \\ Cosmology, Stanford University, Stanford, CA 94305, USA E-mail: \\ tporter@stanford.edu
}

\section{Guôlaugur Jóhannesson}

Science Institute, University of Iceland, IS-107 Reykjavik, Iceland and AlbaNova Univ. Center Nordita, Roslagstullsbacken 23, SE-106 91 Stockholm, Sweden

E-mail: gudlauguehi.is

\section{Igor V. Moskalenko}

W. W. Hansen Experimental Physics Laboratory and Kavli Institute for Particle Astrophysics and Cosmology, Stanford University, Stanford, CA 94305, USA

E-mail: imosestanford.edu

The interstellar radiation field (ISRF) of the Milky Way is the result of emission by stars and re-processing of the starlight by dust in the interstellar medium. The ISRF is an essential input to cosmic-ray (CR) propagation codes for calculating the energy losses by leptons from inverse Compton scattering, and the resulting interstellar $\gamma$-ray emission. To date, models for the ISRF have used a 2D Galactocentric cylindrical symmetric approximation, which neglects details of Galactic structure such as spiral arms and the asymmetric stellar bulge. In this contribution 3D spatial models for the ISRF are presented that incorporate such spatial elements. Because there is no consensus on the geometry of these structures the calculations presented in this contribution employ descriptions for the stellar luminosity and dust distribution taken from the literature and use the Fast Radiation Numerical Kalculation for Interstellar Emission (FRaNKIE) code to generate corresponding spectral intensity distributions throughout the Galaxy. Locally the models fairly successfully reproduce near- to far-infrared data, despite having distinctly different stellar and dust density distributions. Elsewhere in the Galaxy the 3D intensity distributions reflect the differences due to the input stellar and dust density distributions between the models.

35th International Cosmic Ray Conference - ICRC2017

10-20 July, 2017

Bexco, Busan, Korea

${ }^{*}$ Speaker. 


\section{Introduction}

The ISRF is the result of emission by stars, and the scattering, absorption, and re-emission of absorbed starlight by dust in the ISM. It is a critical component for modelling $\mathrm{CR}^{ \pm}$propagation because the spatial distribution of the ISRF spectral intensity determines the energy losses via inverse Compton (IC) scattering, and the ensuing $\gamma$-ray emissions by the same process. Early models for the ISRF were motivated to enable predictions of the Galactic IC emission at $\mathrm{MeV}$ $\gamma$-ray energies [1, 2, 3]. Mathis et al. [4] (hereafter, MMP83) presented calculations of the ISRF from UV to far-infrared wavelengths at a few locations with a model that has been widely used. Bloemen [5] extended this work to study the IC emission in the context of COS-B data, but noted the model shortcomings toward the inner Galaxy because of the artificial cut-off for $R \lesssim 3 \mathrm{kpc}$ in the stellar spatial distribution. Chi \& Wolfendale [6] augmented the MMP83 stellar model with the dust emission results described by Cox [7] and re-calculated the spatial distribution of the ISRF energy density. This work was used by the EGRET team as input to their modelling of the highenergy interstellar $\gamma$-ray emission [8, 9]. Strong et al. [10] employed stellar population models [11] and dust emissivities derived from $C O B E / \mathrm{DIRBE}$ data [12] to re-evaluate the ISRF energy density distribution.

Despite the increasing sophistication of the inputs these early calculations were not selfconsistent because they did not couple the starlight absorption by dust with re-emission. Porter \& Strong [13] describe the first work to combine the stellar emission with a self-consistent dust scattering/absorption/reemission radiation transfer calculation using the $F R a N K I E^{1}$ code. Porter et al. [14] extended the calculations to produce also the spatially varying anisotropic intensity of the ISRF using a 2D stellar and dust density distribution. The Fermi-LAT team [15] used a revision of the Porter et al. [14] work for the study of high-energy $\gamma$-ray interstellar emission models calculated using the GAL$P R O P$ CR propagation code. The spectral intensity distribution used for this latter work represents the latest publicly available model for the ISRF that is distributed from the GALPROP website ${ }^{2}$.

The Galactic structure is spatially complex with spiral arms (e.g., [16]), a central region that is dominated by a bulge/bar complex (e.g., [17, 18]), and warped stellar/dust discs [19]. Sophisticated 3D models have been developed for the spatial densities of stellar populations [20,11]. But their emphasis is on calculating counts of stars as observed from Earth. Three-dimensional spatial models that include asymmetric elements, such as a central bulge/bar, spiral arms, and warped stellar/dust discs, have been fit to infrared data [21] but they obtain only emissivities and do not make radiation transfer calculations. The most comprehensive 3D modelling to-date [22] (hereafter, R12) uses the SKY model [11] as input to the HYPERION code [23] to self-consistently calculate predictions for local observed intensities from near- to far-infrared wavelengths. R12 produced an optimised version of the SKY model that is consistent with Spitzer and IRAS data in a narrow band of latitude about the Galactic plane for longitudes $-60^{\circ} \leq l \leq 60^{\circ}$. However, the spectral intensity was only evaluated locally for comparison with the data.

There is no unique description for the Galactic structure even with the diversity of models so far employed to fit for the different spatial distributions of stars and dust in the Galaxy. A hybrid combining different spatial elements and their parameters from the collection studied in the

\footnotetext{
${ }^{1}$ Fast Radiative Numerical Kalculation for Interstellar Emission.

${ }^{2} \mathrm{http}: / /$ galprop.stanford.edu
} 
literature without a full fitting procedure can lead to inconsistent models because of convariance between the presence of individual elements and their derived parameters, particularly for the major components such as the stellar disc, arms, and bulge. Because the purpose of this contribution is to make the first steps to incorporating 3D spatial structure into the ISRF models employed by CR propagation and interstellar emissions codes like GALPROP such an optimisation is not made. Instead two models for the stellar and dust distributions are chosen from the literature: the R12 model described above and that of Freudenreich [19] (hereafter, F98). They employ different spatial densities for both the stars and dust but produce very similar intensities to the data for nearto far-infrared wavelengths at the Solar system location (see below). The spatial differences can be summarised as the former model has an axisymmetric bulge and smooth disc, and major spiral arms and local arm segments near the Solar system, while the latter has a non-axisymmetric bulge and smooth disc; details of each are given in the respective references and a forthcoming paper (Porter et al., in press).

The FRaNKIE code is used to calculate the Galaxy-wide spectral intensity distribution for the R12 and F98 models, which is necessary for the lepton IC energy losses and $\gamma$-ray production. Its solution method for the radiation transport has evolved from the ray-tracing method initially employed by [13] to using simulation techniques similar to other Monte Carlo radiation transfer codes (e.g., $[22,24])$ where luminosity packets are injected according to user-specified spatial and spectral distributions, and the propagation history of each packet is traced to produce observables that are recorded at observer locations. The propagation includes self-consistent scattering, absorption, and re-emission by dust, the optical properties and spatial distributions of which are also user-specified. A recent description of the code is provided by Porter et al. [25] with further developments covered elsewhere (Porter et al., in prep.).

\section{Calculations and Results}

The simulation volume for the radiation transfer calculations is a box with dimensions $\pm 15 \mathrm{kpc}$ for the $X, Y$ coordinates and $\pm 3 \mathrm{kpc}$ for the $Z$ coordinate because this effectively encapsulates all of the input stellar luminosity and dust for the R12 and F98 models, and hence ensures that computation resources are not wasted for regions that contribute negligibly to the spectral intensity distribution. A Cartesian grid is used to segment the simulation volume. The $X, Y$ coordinates have regular spacing $\Delta X, Y=0.125 \mathrm{kpc}$, while the $Z$ coordinate uses a logarithmic spacing with 25 bins covering 0.001 to $3 \mathrm{kpc}$ plus an additional linear bin for that closest to the Galactic midplane (52 in total). The wavelength grid spans $0.0912-10000 \mu \mathrm{m}$ with 256 logarithmically spaced bins.

To record the spectral intensity distribution a cylindrical grid is used because of the radial and angular dependence of the spatial densities for the R12 and F98 models. Any choice of grid spacing is a compromise balancing computational resources and overall accuracy. The following grid is found to provide adequate sampling for the typical sizes of simulation volumes used for GALPROP CR propagation and interstellar emission calculations (halo sizes $\sim 4-10 \mathrm{kpc}$ perpendicular to the Galactic plane and maximum $X, Y$ boundaries $\sim 20 \mathrm{kpc}$ ). The spacing is variable in Galactocentric radius with $\Delta R=0.1 \mathrm{kpc}$ near the GC, then $\Delta R=0.25 \mathrm{kpc}$ to the Solar circle, $\Delta R=1 \mathrm{kpc}$ beyond to $15 \mathrm{kpc}$, and $\Delta R=5 \mathrm{kpc}$ beyond that to $R=30 \mathrm{kpc}$. Azimuthally the spacing is at $\Delta \phi=10^{\circ}$. The $Z$-coordinates for the camera locations are $\pm 20, \pm 10, \pm 5, \pm 2, \pm 1, \pm 0.5, \pm 0.25, \pm 0.1$, and $0 \mathrm{kpc}$. 
Each ISRF model calculation (R12, F98) uses $5 \times 10^{8}$ luminosity packets with HEALPix [26] Nside $=8$ maps $^{3}$ to determine the Galaxy-wide spectral intensity distributions. These statistics are sufficient because integrating over the ISRF spectral intensity when determining the $e^{ \pm}$energy losses and IC emissivities smooths any artifacts due to Monte Carlo noise. However, for the data/model comparison for the line profiles shown below higher statistic runs are made using $5 \times 10^{10}$ luminosity packets with HEALPix Nside $=1024$ maps. Even with the higher statistics runs there is unavoidable Monte Carlo noise typically where the intensities are lowest.
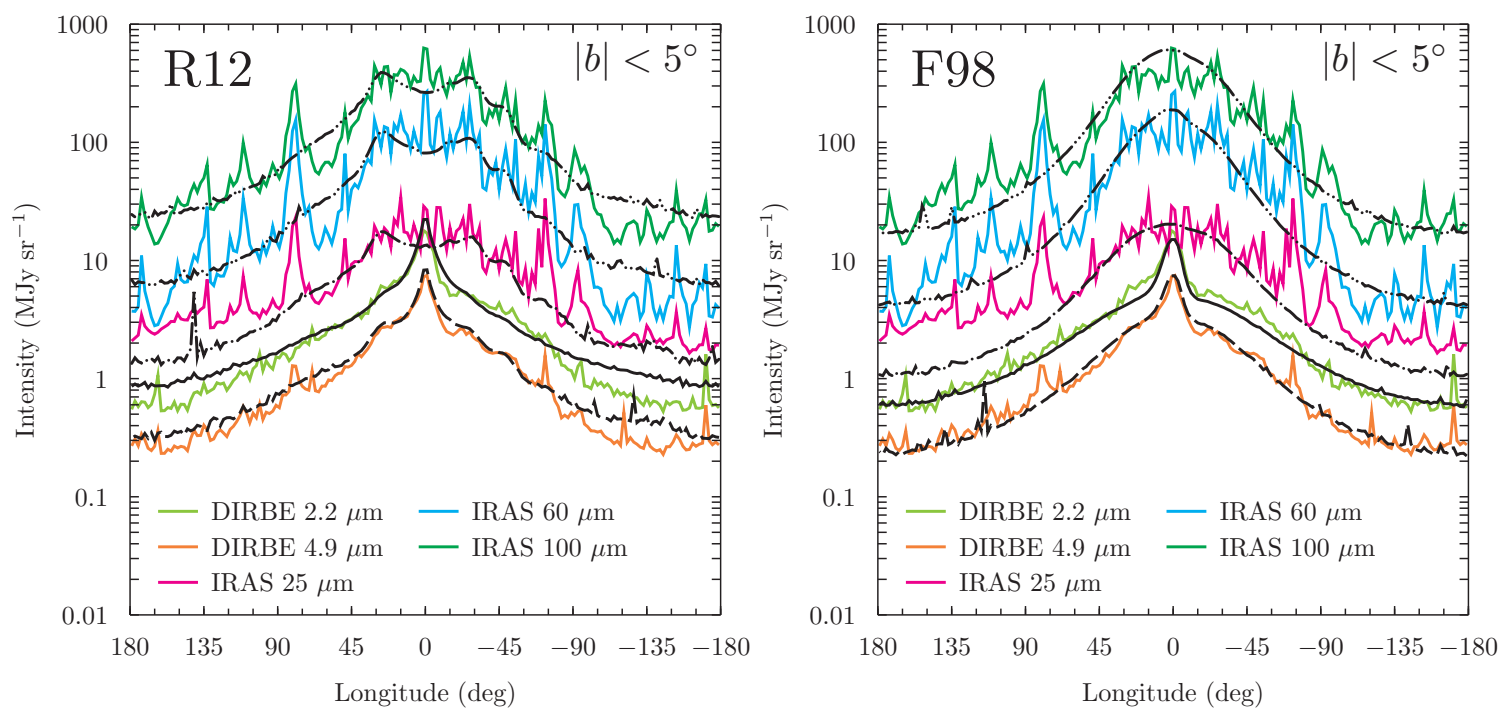

Figure 1: Longitude profile averaged over $-5^{\circ}<b<b^{\circ}$ for the modified SKY (left, R12) and COBE/DIRBE bar/disc (right, F98) models, respectively. Coloured curves show the data for $C O B E / \mathrm{DIRBE}$ and reprocessed $I R A S$, while black lines show the model predictions convolved with the instrumental bandpass overlaid on the data for $C O B E / D I R B E 2.2 \mu \mathrm{m}$ (solid), $4.9 \mu \mathrm{m}$ (long-dashed), and IRAS $25 \mu \mathrm{m}$ (long-dash-dot), $60 \mu \mathrm{m}$ (long-dash-dot-dot), and $100 \mu \mathrm{m}$ (long-dash-triple-dot) bands, respectively.

Figure 1 shows the predicted longitude profiles for latitudes $-5^{\circ}<b<5^{\circ}$ for the R12 (left) and F98 (right) models overlaid with the data from $C O B E / \mathrm{DIRBE}^{4}$ and the reprocessed $I R A S^{5}$ survey. The all-sky model intensity maps are convolved with the instrumental band passes and point spread functions, then averaged over the latitude band to construct the profiles. The models use density distributions for the stars and dust that do not distinguish individual sources. Therefore the comparison with data is made without point-source subtraction.

The bulk features of the observed intensity profiles are generally successfully reproduced by each model. The R12 model traces the structure of the data more closely for $-60^{\circ} \leq l \leq 60^{\circ}$ because this is the region that it has been optimised over. Outside of this longitude range the R12

\footnotetext{
${ }^{3}$ GALPROP anisotropic IC $\gamma$-ray calculations have been tested using the R12 model calculated with Nside $=4,8$, and 16 spectral intensity maps to evaluate the best compromise between accuracy, run-time, and disc storage for the ISRF data files. With the Nside $=8$ and 16 resolutions the position-dependent intensity traces asymmetries (by arms, etc.) sufficiently for the $\gamma$-ray calculations. There is no discernable difference for the IC calculations using the Nside $=$ 8 and 16 maps, but the run-time and storage on-disc for the latter is $\sim 4 \times$ higher.

${ }^{4}$ COBE/DIRBE ZSMA maps retrieved from https://lambda.gsfc.nasa.gov/.

${ }^{5}$ IRIS - Improved Reprocessing of the IRAS Survery [27]: https://www.cita.utoronto.ca/ mamd/IRIS/IrisOverview.html.
} 
intensities tend to be higher than the data at near-infrared wavelengths. This is due to the scalelength of the stellar disc, which produces a shallower profile than the data indicate toward the outer Galaxy, and the presence of the local arms that also contribute in this region. The F98 model agrees well with the near-infrared data over all longitudes owing to the smaller radial scale-length for its stellar discs. However, the structure in the data for $-60^{\circ} \leq l \leq 60^{\circ}$ is not reproduced because the spiral arms are treated as an averaged disc component for this model. For the mid- and far-infrared wavebands the better correspondence of the profile structure by the R12 model is again no surprise because it was optimised to reproduce the features in the data tracing the spiral arm tangents. The F98 model adequately traces the intensity profile given its comparative simplicity to the R12 model. Both models under-predict the mid-infrared in the outer Galaxy. For R12 this is most likely due to mismodelling of the stellar luminosity content of the local arms that are an element of this model. For the F98 model the absence of such local features that causes the lower intensity in these regions.

The discrepancies between the R12 and F98 model predictions and local observations are generally minor. Integrating the intensities over the sky produces all-sky averaged values for both models that are close to the data and that are well within the experimental uncertainties for all wavebands, thus showing the general consistency for both R12/F98 calculations with the observations.
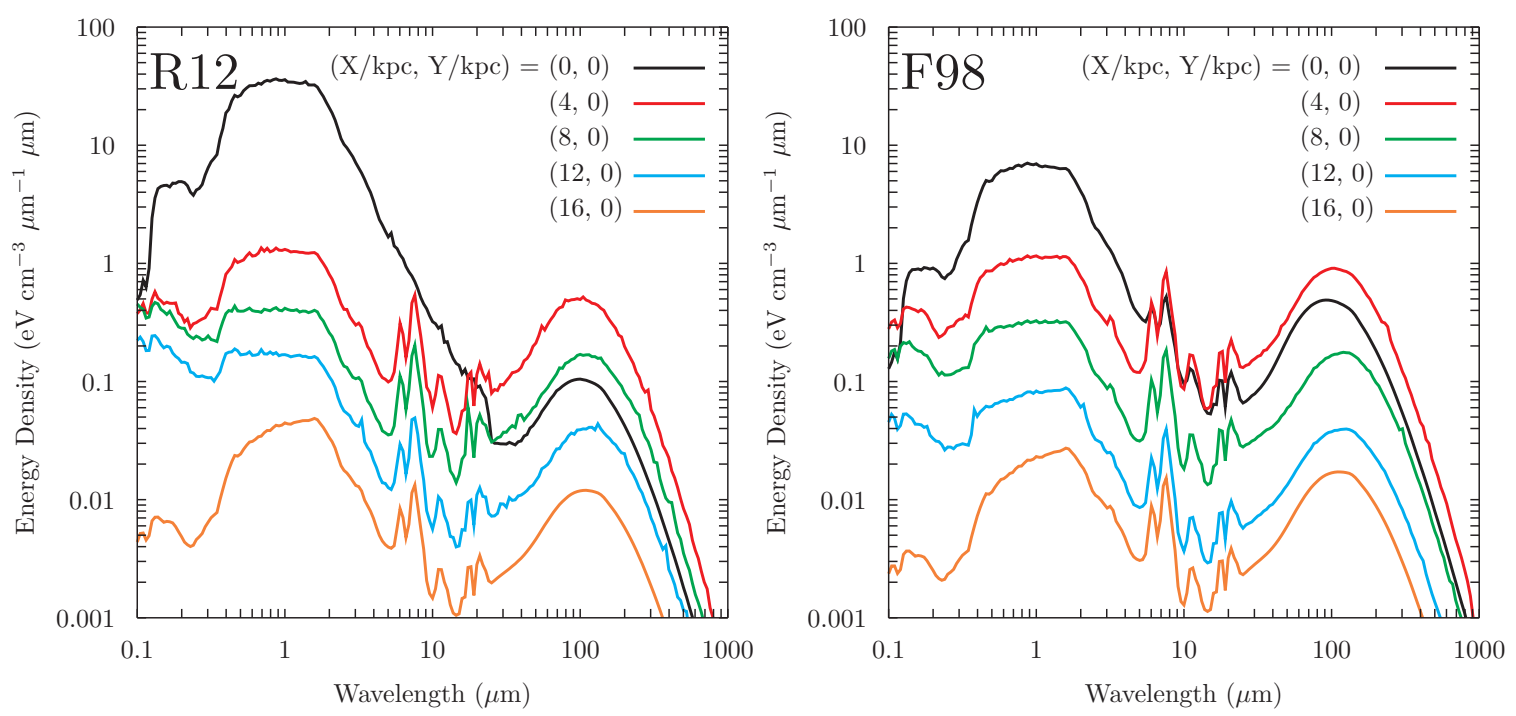

Figure 2: ISRF spectral energy density for the R12 (left) and F98 (right) models, respectively, showing the variation with positive $X$ coordinate in the Galactic plane.

While the predicted intensities locally are very similar those elsewhere in the Galaxy can vary considerably between the R12 and F98 models. Figure 2 shows the spectral energy density (SED) variation with $X$ coordinate in the Galactic plane. Near the GC the R12 model is considerably more intense at UV/optical to near-infrared wavelengths compared to F98. This comes from several differences: the R12 model has a stellar disc that continues toward the GC, while the F98 model has a holed stellar disc; the R12 bulge luminosity is higher than that for the F98 model; and the hole in the R12 dust disc has a much larger radius than that of the F98 model, consequently the absorption near the GC for the R12 model is much lower. However, the general trend of a shift in the peak of the far-infrared emission to shorter wavelengths from the intense radiation field over 

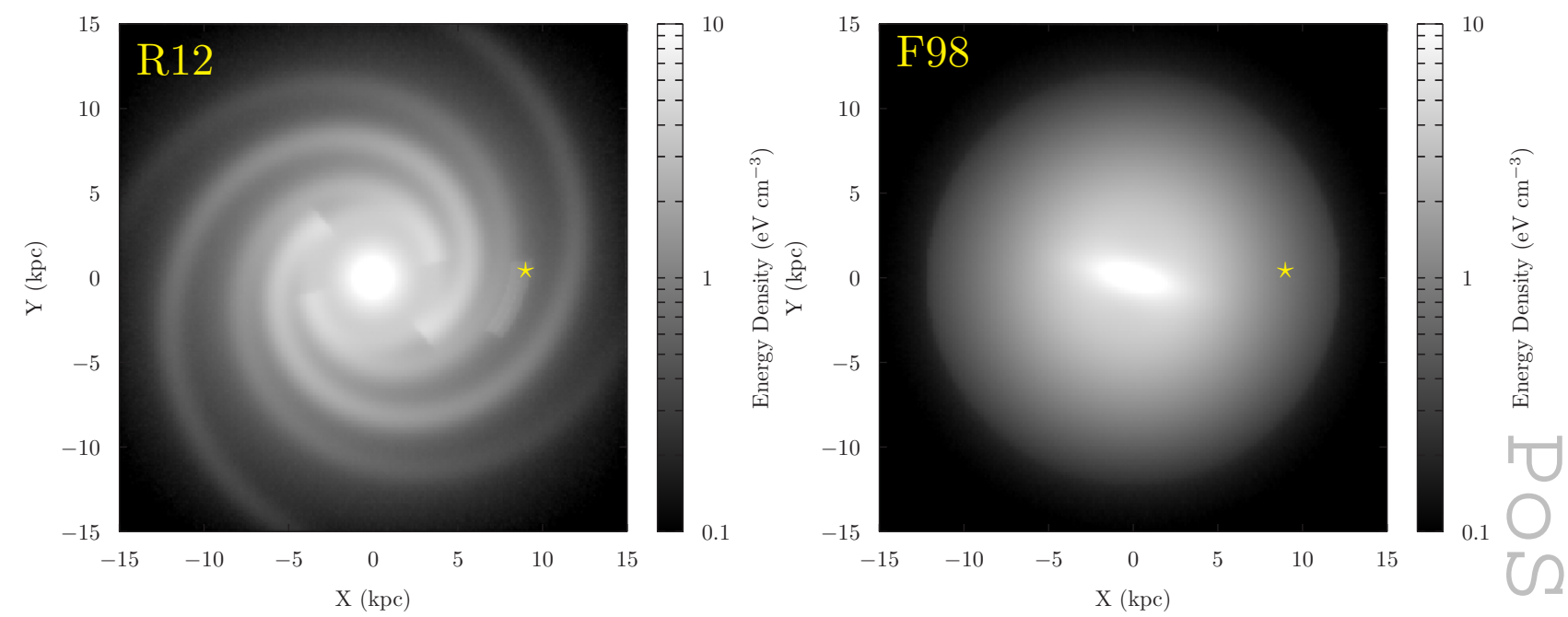

Figure 3: Integrated ISRF energy densities in the Galactic plane for the R12 (left) and F98 (right) models, respectively. The yellow star marks the location of the Solar system for each. Note that the energy density saturates the scale in and about the GC for both models.

the inner Galaxy is present for both R12 and F98 models independent of the dust density there. The other major difference is the spatial variation of the SED from far-UV to $\sim 0.5 \mu \mathrm{m}$ wavelengths that reflects how the early-type stars are modelled. The variation of the UV and optical spectral intensity for R12 model with $X$ outside the region about the GC is driven predominantly by the crossing of the various arms as $X$ increases out to $\sim 12 \mathrm{kpc}$. In particular, for the R12 model the locations shown in the figure sample inter-arm $(X=4 \mathrm{kpc})$ and in-arm $(X=8,12 \mathrm{kpc})$ regions. For the F98 model such variations are absent because the young stars in the arms are treated using spatial averaging.

The variation of the wavelength-integrated SED predicted for the R12 and F98 models across the Galaxy at the mid-plane is shown in Fig. 3. The R12 model produces a more structured spatial distribution for the energy density with features related to the stellar luminosity model clearly evident. The variation of the SED shown in Fig. 2 (left) from the crossing of inter-arm/arm regions with $X$-coordinate is also readily understood where the maxima of emission are clearly visible near $X \sim 8$ and $\sim 12 \mathrm{kpc}$, respectively. For the F98 model the asymmetric bulge produces an elongated region of high energy density dominating the inner Galaxy region. Either side of the minor axis of the bulge are shallow minima in the energy density distribution, and outside of $\sim 4 \mathrm{kpc}$ about the GC the stellar disc is the only significant contributer.

The spatial variation of the SED outside of the Galactic plane (not shown) is most sensitive to the details of the stellar luminosity and dust model for $|Z| \lesssim 1-5 \mathrm{kpc}$, with the effect dependent on position relative to the GC. Close to the GC the intensities of the respective models differ the most but become comparable for $|Z| \simeq 5 \mathrm{kpc}$, while toward the outer Galaxy they are similar for somewhat lower height $|Z| \simeq 1 \mathrm{kpc}$. The fall-off of the intensity with $Z$-coordinate is approximately logarithmic for both models. At a distance $\sim 10 \mathrm{kpc}$ above the plane near the GC the energy density for either model is similar to that of the $\mathrm{CMB}$, and at a distance $\sim 20 \mathrm{kpc}$ becomes comparable that of the extragalactic background light [28]. 
While both R12 and F98 models provide similar descriptions of the data there are improvements possible, in particular optimisation for the parameters of the spatial distributions for the arms, and ellipsoidal bulge/bar, which will be addressed in subsequent work. Nevertheless, the Galaxy-wide R12 and F98 spectral intensity distributions calculated in this paper represent the first such 3D spatial models that can be used to calculate $\mathrm{e}^{ \pm}$energy losses and high-energy $\gamma$-ray emissions by the IC process from the Milky Way. They also provide a useful quantification of the ISRF spectral energy density spatial variations, particularly toward the inner Galaxy where the spatial densities for the stars and dust used by the models are most uncertain. The direct application to interpreting high-energy $\gamma$-ray data with 3D CR source and ISRF spatial density models will be described at the meeting [29]. The data files for both 3D ISRF models will be made available from the GALPROP website and can be used with the forthcoming release of the GALPROP code [30].

\section{Acknowledgments}

FRaNKIE and GALPROP development is partially funded via NASA grants NNX13AC47G and NNX17AB48G. Some of the results in this paper have been derived using the HEALPix [26] package.

\section{References}

[1] R. Cowsik and W. Voges, Inverse Compton gamma rays from the galactic disc, in The Context and Status of Gamma-Ray Astronomy (B. G. Taylor, ed.), Nov., 1974.

[2] P. G. Shukla and J. Paul, Gamma-ray production by the inverse Compton process in interstellar space, ApJ 208 (Sept., 1976) 893-899.

[3] G. F. Bignami and G. Piccinotti, Inverse Compton revisited - Galactic far infrared and optical photon fields, high energy electrons and medium energy gamma rays, A\&A 59 (July, 1977) 233-238.

[4] J. S. Mathis, P. G. Mezger, and N. Panagia, Interstellar radiation field and dust temperatures in the diffuse interstellar matter and in giant molecular clouds, A\&A 128 (Nov., 1983) 212-229.

[5] J. B. G. M. Bloemen, The interstellar radiation field and the production of inverse-Compton gamma rays in the Galaxy, A\&A 145 (Apr., 1985) 391-404.

[6] X. Chi and A. W. Wolfendale, The interstellar radiation field: a datum for cosmic ray physics, Journal of Physics G Nuclear Physics 17 (June, 1991) 987-998.

[7] P. Cox, E. Kruegel, and P. G. Mezger, Principal heating sources of dust in the galactic disk, A\&A 155 (Feb., 1986) 380-396.

[8] D. L. Bertsch et al., Diffuse Gamma-Ray Emission in the Galactic Plane from Cosmic-Ray, Matter, and Photon Interactions, ApJ 416 (Oct., 1993) 587.

[9] S. D. Hunter et al., EGRET Observations of the Diffuse Gamma-Ray Emission from the Galactic Plane, ApJ 481 (May, 1997) 205-240.

[10] A. W. Strong, I. V. Moskalenko, and O. Reimer, Diffuse Continuum Gamma Rays from the Galaxy, ApJ 537 (July, 2000) 763-784, [astro-ph/9811296].

[11] R. J. Wainscoat et al., A model of the 8-25 micron point source infrared sky, ApJS 83 (Nov., 1992) 111-146. 
[12] T. J. Sodroski et al., A Three-dimensional Decomposition of the Infrared Emission from Dust in the Milky Way, ApJ 480 (May, 1997) 173-187.

[13] T. A. Porter and A. W. Strong, A new estimate of the Galactic interstellar radiation field between 0.1um and 1000um, International Cosmic Ray Conference 4 (2005) 77, [astro-ph/ 0507119 ].

[14] T. A. Porter et al., Inverse Compton Origin of the Hard X-Ray and Soft Gamma-Ray Emission from the Galactic Ridge, ApJ 682 (July, 2008) 400-407, [arXiv: 0804.1774 ].

[15] M. Ackermann et al., Fermi-LAT Observations of the Diffuse $\gamma$-Ray Emission: Implications for Cosmic Rays and the Interstellar Medium, ApJ 750 (May, 2012) 3, [arXiv: 1202 .4039].

[16] J. P. Vallée, Catalog of Observed Tangents to the Spiral Arms in the Milky Way Galaxy, ApJS 215 (Nov., 2014) 1, [arXiv:1409.4801].

[17] P. L. Hammersley et al., Detection of the old stellar component of the major Galactic bar, MNRAS 317 (Sept., 2000) L45-L49, [astro-ph/ 0007232 ].

[18] R. A. Benjamin et al., First GLIMPSE Results on the Stellar Structure of the Galaxy, ApJL 630 (Sept., 2005) L149-L152, [astro-ph/ 0508325$].$

[19] H. T. Freudenreich, A COBE Model of the Galactic Bar and Disk, ApJ 492 (Jan., 1998) 495-510, [astro-ph/9707340].

[20] A. Robin and M. Creze, Stellar populations in the Milky Way - A synthetic model, A\&A 157 (Mar., 1986) 71-90.

[21] R. Drimmel and D. N. Spergel, Three-dimensional Structure of the Milky Way Disk: The Distribution of Stars and Dust beyond $0.35 R_{\text {solar }}$, ApJ 556 (July, 2001) 181-202, [astro-ph/0101259].

[22] T. P. Robitaille et al., A self-consistent model of Galactic stellar and dust infrared emission and the abundance of polycyclic aromatic hydrocarbons, A\&A 545 (Sept., 2012) A39, [arXiv:1208.4606].

[23] T. P. Robitaille, HYPERION: an open-source parallelized three-dimensional dust continuum radiative transfer code, A\&A 536 (Dec., 2011) A79, [arXiv:1112.1071].

[24] P. Camps and M. Baes, SKIRT: An advanced dust radiative transfer code with a user-friendly architecture, Astronomy and Computing 9 (Mar., 2015) 20-33, [arXiv:1410.1629].

[25] T. Porter, G. Johannesson, and I. V. Moskalenko, The FRaNKIE code: a tool for calculating multi-wavelength interstellar emissions in galaxies, PoS ICRC2015 (2016) 908.

[26] K. M. Górski et al., HEALPix: A Framework for High-Resolution Discretization and Fast Analysis of Data Distributed on the Sphere, ApJ 622 (Apr., 2005) 759-771, [a st ro-ph/ 0409513 ].

[27] M.-A. Miville-Deschênes and G. Lagache, IRIS: A New Generation of IRAS Maps, ApJS 157 (Apr., 2005) 302-323, [astro-ph/ 0412216 ].

[28] M. G. Hauser and E. Dwek, The Cosmic Infrared Background: Measurements and Implications, ARA\&A 39 (2001) 249-307, [astro-ph/ 0105539 ].

[29] T. A. Porter, G. Johannesson, and I. V. Moskalenko, High-Energy $\gamma$-rays from the Milky Way: Three-Dimensional Spatial Models for the Cosmic-Ray and Radiation Field Densities, These Proceedings (July, 2017).

[30] I. V. Moskalenko et al., GALPROP Code for Galactic Cosmic Ray Propagation and Associated Photon Emissions, These Proceedings (July, 2017). 